\title{
Interbranch Parametric Oscillation in the Weak Coupling Regime for Semiconductor Vertical Multimicrocavities
}

\author{
D. Taj, ${ }^{*}$ T. Lecomte, C. Diederichs, Ph. Roussignol, C. Delalande, and J. Tignon \\ Laboratoire Pierre Aigrain, Ecole Normale Suprieure, \\ UMR 8551 CNRS, UPMC Univ. Paris 675005 Paris, France
}

\begin{abstract}
We develop a model able to properly describe a new variety of Optical Parametric Oscillations (OPO's) in some recently realized vertical semiconductor multimicrocavities. Our model shows that, contrary to the case of a conventional single microcavity, the OPO can take place even when the system is brought in the weak light-matter coupling. Our predictions agree qualitatively with experiments, they show some OPO configurations which are peculiar of the weak-coupling and suggest some new experiment. The model clearly shows the need to have a better understanding of Optical Parametric Oscillations in this regime.
\end{abstract}

PACS numbers: 42.65.Yj, 71.36.+c, 05.30.Jp

Optical Parametric Oscillation (OPO) is a nonlinear process that involves coherent oscillations among an excited pump mode and two other modes called signal and idler, generally shifted in energy with respect to the pump [1, 2], thus allowing generation of new frequencies, as well as of twin or entangled pairs of photons for quantum information applications such as quantum cryptography $[3,4]$. Up to now, OPO has been studied in semiconductors microcavities only in the strong coupling regime, as the S-shaped lower polariton branch can allow for the phase matching conditions (ph.m.c.), which are energy and momentum conservation, when the pump is placed at the so called magic angle [5-8]. However, this has several important drawbacks, as discussed in [9]. One of them is for example that the ph.m.c. cannot be satisfied when the system is brought in the weak excitonphoton coupling by say, increasing temperature or pump excitation power: this is at variance with the need of finally realizing an all-semiconductor oscillator able to integrate into nowadays technologies and at the same time to operate at room temperature.

To overcome these intrinsic limitations, some new monolithic structures, called vertical multimicrocavities, have been recently designed [9]. Within these structures, two new types of OPO can be in principle investigated (see Figure 1): thanks to the degeneracy splitting of the coupled cavity modes, a possibility arises to obtain three polaritonic modes at normal incidence, splitted in energy, that can parametrically oscillate, once the ph.m.c. are reached by suitable tuning of the polariton energies (vertical $O P O$ ). Another setup can be obtain by exciting a pump polariton at normal incidence in the second polariton branch, which in turn oscillates with signal and idler polaritons at pump energy and opposite angle in the first branch (horizontal OPO).

Both types of OPO's could in principle occur also in the weak-coupling between cavity photons and excitons, as the ph.m.c. could well be satisfied. However, no study has been made theoretically up to now, to understand the OPO's in the foretold regime.

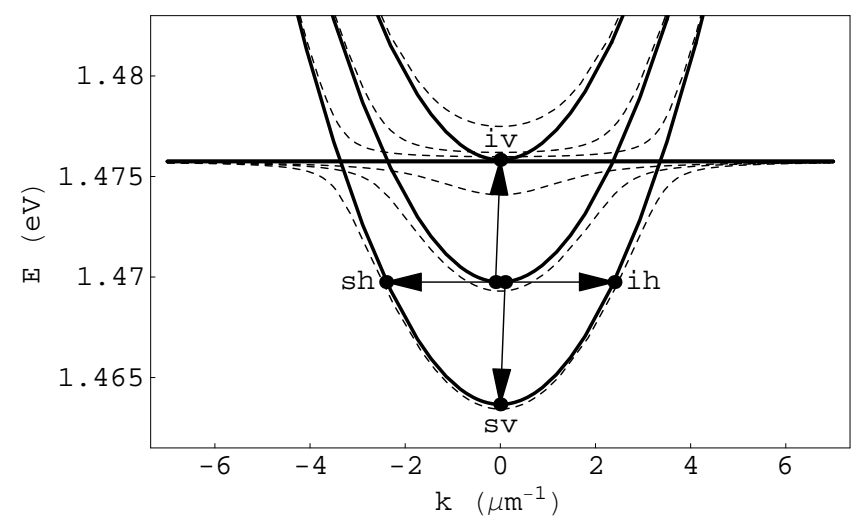

FIG. 1: Typical energy dispersions for a realistic triple microcavity in the weak (thick lines) and strong (thin dashed lines) coupling regime. In this example, all the three excitons are resonant with the third cavity mode at normal incidence, while degeneracy is removed at strong coupling. The arrows represent the two types of OPO studied in this work, in the weak regime: horizontal arrows (signal and idler beams labelled as "sh" and "ih") refer to the horizontal OPO, whereas vertical arrows (signal and idler beams labelled as "sv" and "iv") refer to the vertical OPO.

In this paper we present a theoretical framework able to show an OPO onset in the weak coupling regime, as was indeed observed with considerable amount of evidence in [10] for the case of vertical OPO in triple microcavities. Moreover, our model provides some means for a careful design of the microcavity in the weak coupling, and predicts some phenomena peculiar of the weakcoupling, like resonances in the vertical OPO and pump density dependent photon lifetimes renormalization.

We start considering the usual form of the Hamiltonian that models polariton parametric interactions (see for example [5, 11]), and generalize it to the case of a vertical multimicrocavity with $n$ cavities and $m$ quantum wells $(m \leq n)$ embedded in the cavities. Although, accordingly, we will start from a quantum setting, also for formal simplicity and convenience, our results will be 
classical in nature, and could well be restated in terms of the classical field theory approach in [12]. The main difference with the usual model is a cavity-cavity interaction term, which couples consecutive cavities in the vertical structure and is physically implemented by the cavity coupling Bragg reflectors. The strong coupling between two consecutive cavities is simply modelled by $H^{C C}=\sum_{j=1}^{n-1} g_{j} \sum_{k}\left(\hat{a}_{k}^{j}\right)^{\dagger} \hat{a}_{k}^{j+1}+h . c$., where any of the two consecutive cavity couplings $g_{j}$ is assumed to be real, and $\hat{a}_{k}^{j}$ destroys a photon of planar wavevector $k$. For simplicity will only look at polarization independent processes, although polarization is known to play potentially crucial roles in exciton-polariton microcavities (see for example [13]). Since photons in consecutive cavities are optically coupled, and each of them is only weakly coupled with its cavity embedded 1s-exciton, we can split the full hamiltonian as $H=H_{0}+H_{\text {int }}$. Here $H_{0}=H_{\text {free }}^{C}+H_{\text {free }}^{X}+H^{C C}$ is a sum of the free cavity and 1s-exciton term, plus the foretold cavity-cavity coupling contribution. $H_{\text {int }}=H^{X C}+H^{X X}+H_{\text {sat }}^{X C}$ contains the following interaction terms: $H^{X C}$ is the exciton-photon coupling, with coupling constants $\omega_{j}$ between each cavity photon and its embedded exciton; $H^{X X}$ is the twobody exciton-exciton interaction, with coupling constant $\frac{V_{0}}{2}\left(V_{0}=\frac{6 e^{2} \lambda_{X}}{S \epsilon}, e\right.$ being the electric charge, $\lambda_{X}$ the twodimensional exciton radius, $\epsilon$ the static dielectric constant of the quantum well and $S$ is the macroscopic quantisation area); $H_{\text {sat }}^{X C}$ is the hanarmonic exciton saturation term (coupling $n_{\text {sat }}=\frac{7}{16 \pi \lambda_{X}^{2}}$ ).

Stating that consecutive cavities are strongly coupled amounts to say that there are stable modes among the cavities that diagonalize the free cavity term together with the cavity-cavity coupling $H_{\text {free }}^{C}+H^{C C}$ : if the diagonalising unitary matrix is $U_{j}^{j^{\prime}}(k)$, then we can interpret the new operators $\hat{A}_{k}^{j}=U_{j^{\prime}}^{j}(k) \hat{a}_{k}^{j^{\prime}}$ as destruction operators of collective cavity photon modes which are delocalised over the entire multimicrocavity structure. Then each mode $\hat{A}_{k}^{j}$, with bare energy $E_{C}^{j}(k)$, will be coupled to every 1s-exciton mode through the exciton-photon coupling term $H^{X C}=\sum_{j=1}^{n} \sum_{j^{\prime}=1}^{m} \sum_{k} \Omega_{j}^{j^{\prime}}(k)\left(\hat{A}_{k}^{j}\right)^{\dagger} \hat{b}_{k}^{j^{\prime}}+h . c$. , where we have defined $\Omega_{j}^{j^{\prime}}(k) \equiv \omega_{j^{\prime}} U_{j}^{j^{\prime}}(k)$ and $\hat{b}_{k}^{j^{\prime}}$ is the exciton destruction operator. Similar "non-locality" in the interaction mechanism between excitons and collective photon modes also appears for saturation term $H_{\text {sat }}^{X C}$. Despite the fact that the exciton-photon coupling has become complex and wavevector dependent, it still conserve the planar wavevector. We note that the light-matter coupling now involves two basic quantities, the excitoncavity photon coupling $\omega$ and the matrix element $U_{j}^{j^{\prime}}(k)$, which represents the amplitude of the $j$-th photon collective mode at the $j^{\prime}$-th cavity. This implies that the efficiency of the OPO among collective photon modes will depend upon both such parameters, whom optimization would then become important for a good design of the structure.

In what follows we will focus on the case of the so called horizontal OPO, a priori possible when $m \geq 1$ and $n \geq 2$, while keeping in mind that the treatment of the vertical OPO follows exactly the same lines. To simplify notation we will take $m=1$ quantum well (so we will drop the exciton index $j^{\prime}$ ), embedded say in the first cavity, and $n=2$ cavities, thus linked by only one cavity coupling Bragg mirror. To further simplify, in dealing with the excitonic modes we will only consider excitonexciton interaction brought by $H^{X X}$, and just mention that the treatment of the saturation term $H_{s a t}^{X C}$ is completely analogous.

As stated in the introduction for the horizontal process, we now select the basic interaction process as given by the channel $\{0,0\} \rightarrow\{\kappa,-\kappa\}$. Actually, the ph.m.c.'s would still only fix the norm of the wavevector $\kappa$, so that in principle the horizontal OPO could take place among any two opposite beams in the ring of planar wavevectors of norm $\|\kappa\|$. However, as shown experimentally in [14], because of elastic strain relaxation in the DBR's only few discrete modes in the ring will be involved in the process, often only two, so that from now we will be safely consider a given, fixed wavevector $\kappa$.

First, we add a damping/dephasing process governed by damping constant $\gamma_{X}$ and $\gamma_{C}^{i}$, for the excitonic and the i-th cavity modes respectively; we also add a pump excitation driving field $f_{p}$, resonant with the second cavity mode at normal incidence. Then, by denoting mean field expectations with $x=\langle\hat{x}\rangle$, where $\hat{x}$ can be $\hat{A}$ or $\hat{b}$, we easily write down the mean field equations for the cavity modes:

$$
\left\{\begin{array}{l}
i \hbar \partial_{t} A_{0}^{2}=\left(E_{C}^{2}(0)-i \gamma_{C}^{2}\right) A_{0}^{2}+\Omega_{2}(0) b_{0}+f_{p} \\
i \hbar \partial_{t} A_{ \pm \kappa}^{1}=\left(E_{C}^{1}( \pm \kappa)-i \gamma_{C}^{1}\right) A_{ \pm \kappa}^{1}+\Omega_{1}( \pm \kappa) b_{ \pm \kappa}
\end{array}\right.
$$

At this point, we formally solve the mean field equations of the exciton modes by recursion. That is, we say that the solutions are fixed points of the converging sequence of the complex-valued $l$-th iterate $\left\{b_{0}^{(l)}, b_{\kappa}^{(l)}, b_{-\kappa}^{(l)}\right\}$ as $l \rightarrow+\infty$ : we thus obtain an infinite set of first-order, uncoupled in-homogeneous linear differential equations

$$
\left\{\begin{aligned}
i \hbar \partial_{t} b_{0}^{(l+1)}= & \left(E_{X}(0)-i \gamma_{X}\right) b_{0}^{(l+1)} \\
& +\Omega_{2}(0) A_{0}^{2}+2 V_{0} b_{0}^{(l) *} b_{\kappa}^{(l)} b_{-\kappa}^{(l)} \\
i \hbar \partial_{t} b_{ \pm \kappa}^{(l+1)}= & \left(E_{X}( \pm \kappa)-i \gamma_{X}\right) b_{ \pm \kappa}^{(l+1)} \\
& +\Omega_{1}( \pm \kappa) A_{ \pm \kappa}^{1}+V_{0} b_{\mp \kappa}^{(l) *}\left(b_{0}^{(l)}\right)^{2}
\end{aligned}\right.
$$

with initial condition $b_{k}^{(0)}=0$. This reads like an expansion in powers of the exciton-photon coupling constant $\omega$, and is thus describing in a perturbative fashion a weak coupling regime between photon and exciton, as justified by the absence of a Rabi splitting. The approach is of course perturbative also with respect to the excitonexciton interaction and saturation. 
Since $b_{k}^{(l)}$ will contain factors involving products up to $3^{(l-1)}$ photon mean field expectations, it follows that the second iteration will suffice to grasp the $\chi^{3}$ physics of the OPO in our microcavity. When we substitute the 2-nd iteration exciton operators $b_{k}^{(2)}$ into the Heisenberg equations for the photon modes we get a coupled set of three integro-differential equations for the photon modes in closed form, that is, the set of equations only involves collective photon mean field expectation, and does not involve exciton modes.

In order to do get rid of the memory terms and obtain the OPO's equation it becomes useful to turn to interaction picture, $A_{k}^{i}(t) \equiv e^{\frac{E_{C}^{i}(k) t}{i \hbar}} \tilde{A}_{k}^{i}(t)$, and make a Markov, or slowly varying approximation, on the newly defined, interacting operator $\tilde{A}_{k}^{i}(t)$. This amounts to say that we neglect the time variation of $\tilde{A}_{k}^{i}$ with respect to that of $A_{k}^{i}$, that is, we assume that the time variation brought by the exciton-photon interaction is slow compared to the free energy photons oscillations, in agreement with the hypothesis of weak coupling regime. This approximation allows us to evaluate at current time $t$ each of the three interacting photon operators $\tilde{A}_{k}^{i}$ in the integral kernel.

By doing so, and starting from (1), we find the OPO equations for two pump photons at normal incidence in the second photon branch that are parametrically oscillating with a signal and an idler photon at opposite angles in the first photon branch:

$$
\left\{\begin{array}{l}
i \hbar \partial_{t} A_{0}^{2}=\left(E_{C}^{2}(0)-i \gamma_{C}^{2}\right) A_{0}^{2}+2 E_{i n t}\left(A_{0}^{2}\right)^{*} A_{\kappa}^{1} A_{-\kappa}^{1}+f_{p} \\
i \hbar \partial_{t} A_{ \pm \kappa}^{1}=\left(E_{C}^{1}( \pm \kappa)-i \gamma_{C}^{1}\right) A_{ \pm \kappa}^{1}+E_{i n t}^{*}\left(A_{\mp \kappa}^{1}\right)^{*}\left(A_{0}^{2}\right)^{2} .
\end{array}\right.
$$

Here we have omitted for clarity energy and photon lifetimes renormalization, together with pump blueshift, that we'll consider separately later. Rescaling fields for the quantization area and taking into account also the saturation contribution $H_{\text {sat }}^{X C}$, we compute

$$
E_{\text {int }}=\left(K^{X X} \frac{V_{0}}{2}+K_{\text {sat }}^{X C} \frac{\omega}{n_{\text {sat }}}\right)\left(\Omega_{2}^{\dagger}(0)\right)^{2} \Omega_{1}(\kappa) \Omega_{1}(-\kappa)
$$

with $K^{X X}$ and $K_{\text {sat }}^{X C}$ suitable nonlinear constants. Extensions of this formula to the general case of $n$ cavities and $m$ quantum wells is straightforward. In order to give a better physical understanding of the foretold nonlinear constants, we define the detuning according to $\delta \equiv E_{C}^{2}(0)-E_{X}(0)$, we make the safe approximation $E_{X}(k) \simeq E_{X}(0)$, valid for small wavevectors $k$, and chose the signal and idler mode so that the phase matching conditions hold, which are known (see for example [7]) to enhance the parametric process: this amounts to say that the signal and idler wavevector is chosen to satisfy the energy condition $E_{C}^{1}(\kappa)=E_{C}^{2}(0)$. With this in mind, we compute

$K^{X X}=\frac{1}{2}\left(\frac{1}{\delta^{2}+\gamma_{X}^{2}}\right)^{2}, K_{\text {sat }}^{X C}=\left(\frac{1}{\delta^{2}+\gamma_{X}^{2}}\right)\left(\frac{1}{\delta+i \gamma_{X}}\right)$.

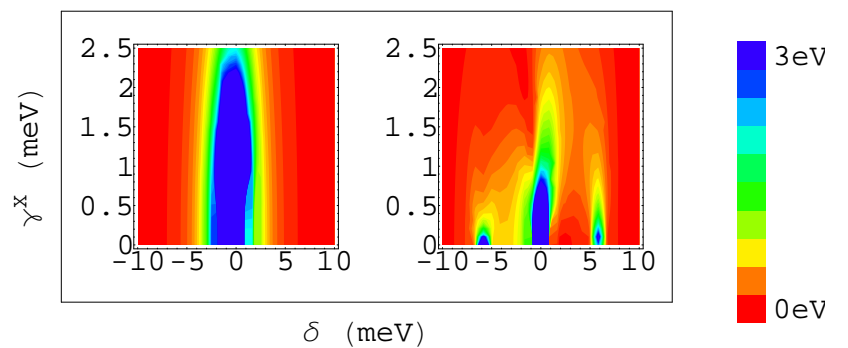

FIG. 2: (Color online) $\left|E_{\text {int }}\right|$ plotted against the detuning $\delta$ and exciton relaxation times $\gamma_{X}$ for the case of horizontal (left) and vertical (right) OPO in the weak coupling.

Needless to say, similar expressions occur also for the vertical OPO, with all the three beams at normal incidence, signal in 1-st branch and idler in the 3-rd, with the only difference that the photon band gap due to the strong cavity-cavity coupling also appears along with the detuning $\delta$.

Moreover, we find energy and photon lifetimes renormalization for the $i$-th collective cavity mode,

$$
\Delta E_{C}^{i}=\frac{\omega^{2}\left(E_{C}^{i}-E_{X}\right)}{\left(E_{C}^{i}-E_{X}\right)^{2}+\gamma_{X}^{2}}, \Delta \gamma_{C}^{i}=\frac{\omega^{2} \gamma_{X}}{\left(E_{C}^{i}-E_{X}\right)^{2}+\gamma_{X}^{2}},
$$

and a pump blueshift of

$$
\left[\frac{V_{0} \omega^{4}}{\left[\delta^{2}+\gamma_{X}^{2}\right]^{2}}+\frac{1}{n_{\text {sat }}} \frac{\omega^{4}}{\delta^{2}+\gamma_{X}^{2}}\left(\frac{1}{\delta+i \gamma_{X}}\right)\right]\left(A_{0}^{2}\right)^{\dagger} A_{0}^{2}
$$

Extension of these formulas to the case of more than one exciton shows that each of the above factors is weighted by $\left(U_{j}^{i}\right)^{\dagger} U_{i}^{j}$, and implies that careful optical design has an important role not only for obtaining high values for the nonlinear OPO constant, but also for energy and lifetimes renormalizations, and blueshift. We also see that if the exciton-exciton interaction brings pure energy blueshifts, the the role of the anharmonic saturation term is to also give rise to pump density dependent photon lifetimes renormalization, thanks to the imaginary term in the above equation: we see here a rich interplay among photon and exciton energies and lifetimes, which in turn are known to trigger crucial effects in semiconductor microcavities [15]. Since the OPO threshold pump power is directly linked to the nonlinear constant $E_{\text {int }}$ through the relation $\left|E_{\text {int }}\right|\left|A_{t h r}\right|^{2}=\gamma_{C}^{1}$, where $\left|A_{t h r}\right|^{2}$ is the threshold pump density, we plot the quantity $\left|E_{\text {int }}\right|$ as a function of detuning $\delta$ and exciton broadening $\gamma_{X}$ for the horizontal and vertical processes for the realistic case of a vertical semiconductor triple microcavity (Figure 2). Blueshift for the pump energy and pump lifetime is displayed in Figure 3 for both types of OPO, in units of pump density.

The plots show that the optimal configuration to trigger the OPO at low thresholds is achieved at nearly zero detunings. However, for the vertical case we see a nonzero 


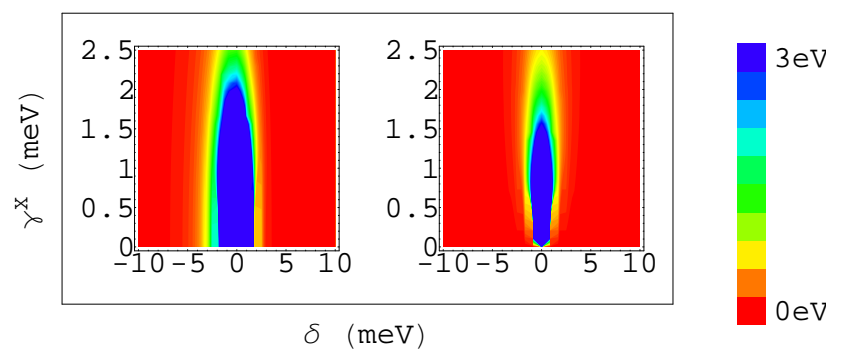

FIG. 3: (Color online) Pump energy and lifetime blueshift in units of pump density plotted against the detuning $\delta$ and exciton relaxation times $\gamma_{X}$ for both horizontal and vertical OPO's.

nonlinear constant also when the exciton is put at resonance with the the signal or the idler mode. This encouraging result explains the experimental observation in [10] of a vertical OPO in the weak coupling where the exciton line is put at resonance with the signal mode, and is peculiar of the weak coupling: in the strong coupling regime, an exciton simply cannot be put at resonance with one polariton mode; if instead it is at resonance with a cavity mode, Rabi splitting occurs that shifts all the energy levels, thus destroying the ph.m.c.'s. The model also suggests that another resonance, namely $\delta \simeq E_{C}^{2}-E_{C}^{3}$, could be available for observing vertical OPO, which in fact wouldn't suffer from the exciton continuum absorption that so highly reduces idler lifetimes at the opposite detuning: this would give hope for comparable signal and idler lifetimes that might allow for quantum correlations and entanglement among twin beams.

After matching our model with the experiment results for the vertical case, we summarize in Figure 4 our predictions on experiments for horizontal OPO's in two different configurations, that is for the case of a triple and a double microcavity. We find a very low threshold for the triple microcavity, $14 \mathrm{KW} / \mathrm{cm}^{2}$ to be compared to $2400 \mathrm{KW} / \mathrm{cm}^{2}$ for the vertical case, and an even smaller one for the double microcavity, $4.4 \mathrm{KW} / \mathrm{cm}^{2}$, due to different optical design, that is, different pump amplitudes in each cavity. The factor of more than $10^{2}$ between the vertical and both the horizontal processes comes from their different setups: putting the exciton at resonance with the pump in the horizontal case results in a far greater nonlinear constant. Even though we found that the horizontal configurations enjoy ultra-low thresholds, which are by themselves difficult to observe experimentally, we are faced with the drawback of very weak signal and idler intensities, as they are proportional to the inverse of the nonlinear constant. In such a configuration, the OPO square root dependence of signal and idler beam intensities could then be hidden by other more important effects, like the Rayleigh scattering, which are known to be linear in the pump excitation power. We note that our predictions for the horizontal OPO don't take into account many body correlations, which could however become important for resonant excitons [16].

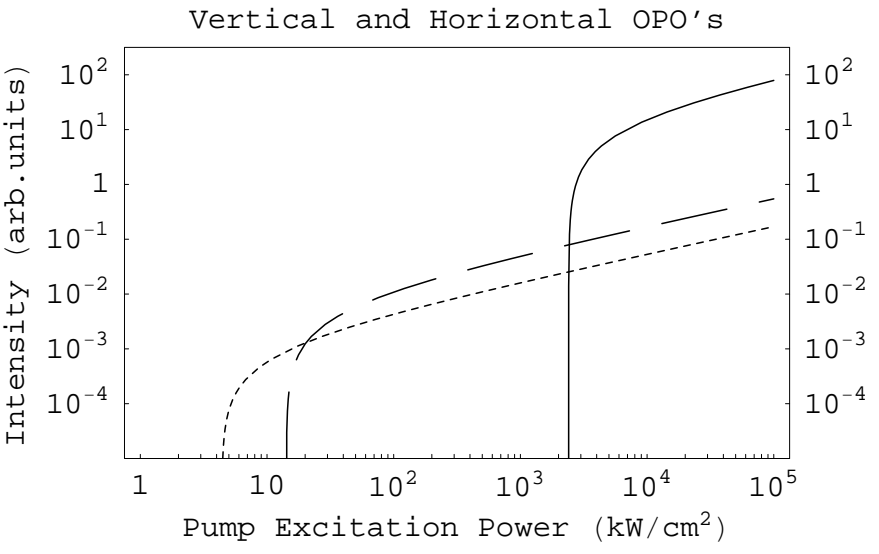

FIG. 4: Signal intensities plotted in logarithmic scale against pump excitation power, for the vertical OPO (continuous line), horizontal OPO in a triple (light dashing) and double (thick dashing) microcavity.

To summarize, we have developed a model able to describe a variety of Optical Parametric Oscillations that occur in vertical semiconductor multimicrocavities in the weak-coupling regime. The exciton energies and lifetimes, together with the detuning, are free parameters of our model, and can be tuned for optimal design. We have shown that within this framework we are able to understand observed experimental data (vertical OPO) and predict OPO's threshold and beam intensities for a variety of other configurations. The model also gives energy and photon lifetimes renormalization, together with pump blueshift, predicts some phenomena peculiar of the weak-coupling and suggests new experiments. Of course, the model doesn't take into account Raylegh scattering, crystal structure, and even the possibility that the exciton could bleach at high temperature and pump power. As such, it demonstrates the urgent need of a more complete formalism to study the rich variety of phenomena occurring in weakly coupled multiple microcavities.

* david.taj@gmail.com

[1] Shen, Y.R. The Principles of Nonlinear Optics (Wiley Inter-Science, Hoboken, 2003)

[2] Armstrong, J.A., Bloembergen, N., Ducuing, J., \& Pershan, P.S., Interaction between light waves in a nonlinear dielectric, Phys.Rev. 127, 1918-1939 (1962)

[3] Jennewein, T., Simon, C., Weish, G., Weinfurter, H. \& Zeilinger, A. Quantum Criptography with Entangled Photons, Phys. Rev. Lett. 84, 4729-4732 (2000)

[4] Gisin, N., Robordy, G., Tittel, W. \& Zbinden, H., Quantum Cryptography, Rev. Mod. Phys. 74, 145-195 (2002)

[5] Ciuti, C., Schwendimann, P. \& Quattropani, A., Theory of polariton parametric interactions in semiconductor mi- 
crocavities, Semicond. Sci. Technol. 18, 279-293 (2003)

[6] Ciuti, C., Schwendimann, P., Deveaud, B. \& Quattropani, A., Theory of the angle-resonant polariton amplifier, Phys. Rev. B 62, R4825 (2000)

[7] Kavokin, A.V., Baumberg, J.J., Malpuech, G. \& Laussy, F.P., Microcavities, Oxford University Press (2007)

[8] Saddavis, P.G. et al. , Angle-resonant stimulated polariton amplifier, Phys. Rev. Lett. 84, 1547 (2000)

[9] Diederichs, C. \& Tignon, J., Design for a triply resonant vertical-emitting micro-optical parametric oscillator, Appl. Phys. Lett. 87, 251107 (2005)

[10] Diederichs, C., Tignon, J., Dasbach, G., Ciuti, C., Lemaître, A., Bloch, J., Roussignol, Ph. \& Delalande, C., Parametric oscillation in vertical triple microcavities, Nature 440 (2006)

[11] Savona, V., Hradil, Z., Quattropani, A., \& Schwendimann, P., Quantum theory of quantum-well polaritons in semiconductor microcavities, Phys. Rev. B 49, 8774 8779 (1994)

[12] Whittaker, D. M., Classical treatment of parametric pro- cesses in a strong-coupling planar microcavity, Phys. Rev. B 63, 193305 (2001)

[13] Kavokin, K. V., Shelykh, I. A., Kavokin, A. V., Malpuech, G. \& Bigenwald P., Quantum Theory of Spin Dynamics of Exciton-Polaritons in Microcavities, Phys. Rev. Lett. 92, 017401 (2004)

[14] Diederichs, C., Taj, D., Lecomte, T., Ciuti, C., Roussignol, P., Delalande, C., Lemaître, A., Largeau, L., Mauguin, O., Bloch, J., Leyder, C., Bramati, A., Giacobino, E., \& Tignon, J., Parametric generation of twin photons in vertical triple microcavities, Comptes Rendus Physique, 8, 10, 1198-1204 (2007)

[15] Whittaker, D. M., What Determines Inhomogeneous Linewidths in Semiconductor Microcavities?, Phys. Rev. Lett. 80, 4791 - 4794 (1998)

[16] Kwong, N.H., Takayama, R., Rumyantsev, I., KuwataGonokami, M. and Binder, R., Third order exciton correlation and nonlinear cavity-polariton effects in semiconductor microcavities, Phys. Rev. B 64, 045316 (2001) 